\title{
Integration and testing of an imaging spectrometer for earth observation
}

T. Peschel, M. Beier, C. Damm, J. Hartung, R. Jende,

S. Müller, M. Rohde, A. Gebhardt, S. Risse

Fraunhofer IOF

I. Walter, I. Sebastian, D. Krutz DLR Opt. Sensors 


\section{Outline}

- Introduction

- Components and pre-integration

- Instrument integration and results

- Summary 


\section{Hyperspectral imaging}

- Image a scene in multiple colors

- Provide additional sensitivity for:

- environmental status

- agriculture

- mineral content ...

- Two basic concepts:

- (conventional) imager + filters

$\Rightarrow$ limited number of channels ( 10..20)

$\Rightarrow$ low to medium spectral resolution

- imaging spectrometer

$\Rightarrow$ high number of channels ( 100..200)

$\Rightarrow$ high spectral resolution

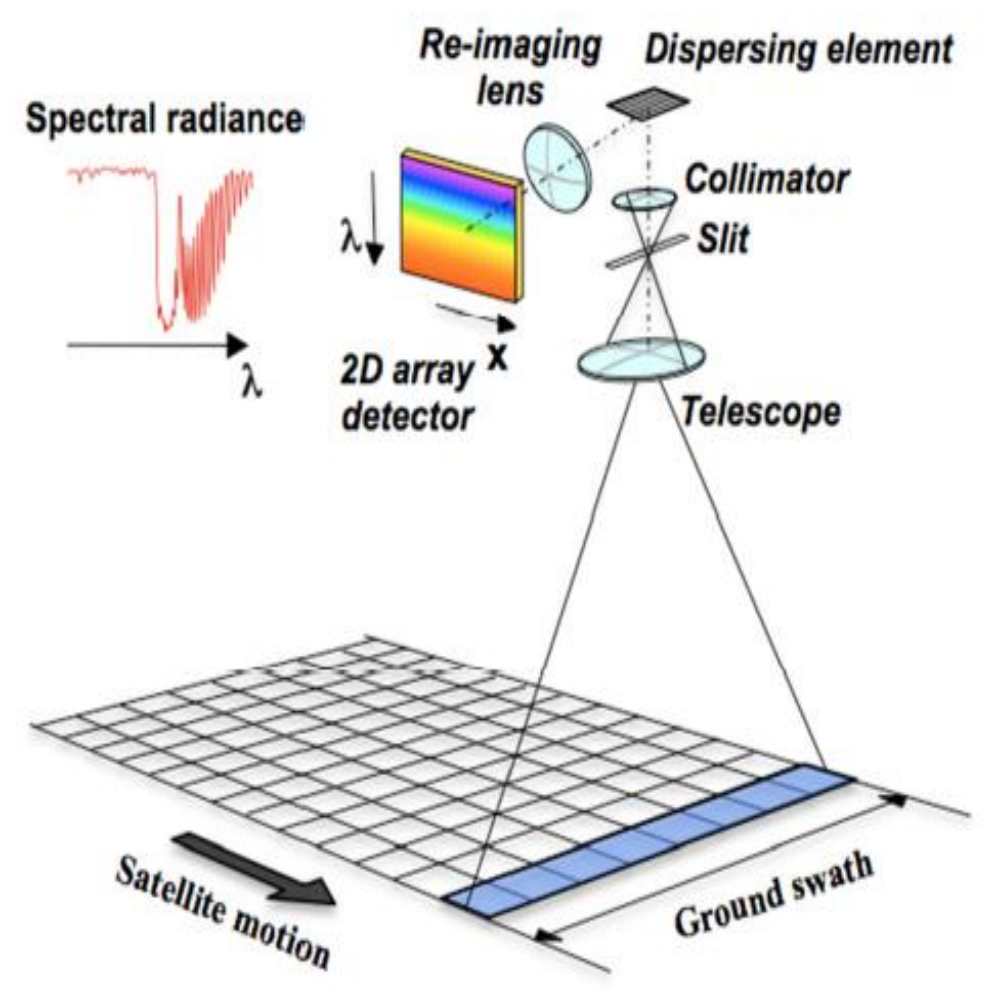

Basic outline of an imaging spectrometer

Source: Kraft e.a., ICSO 2012 


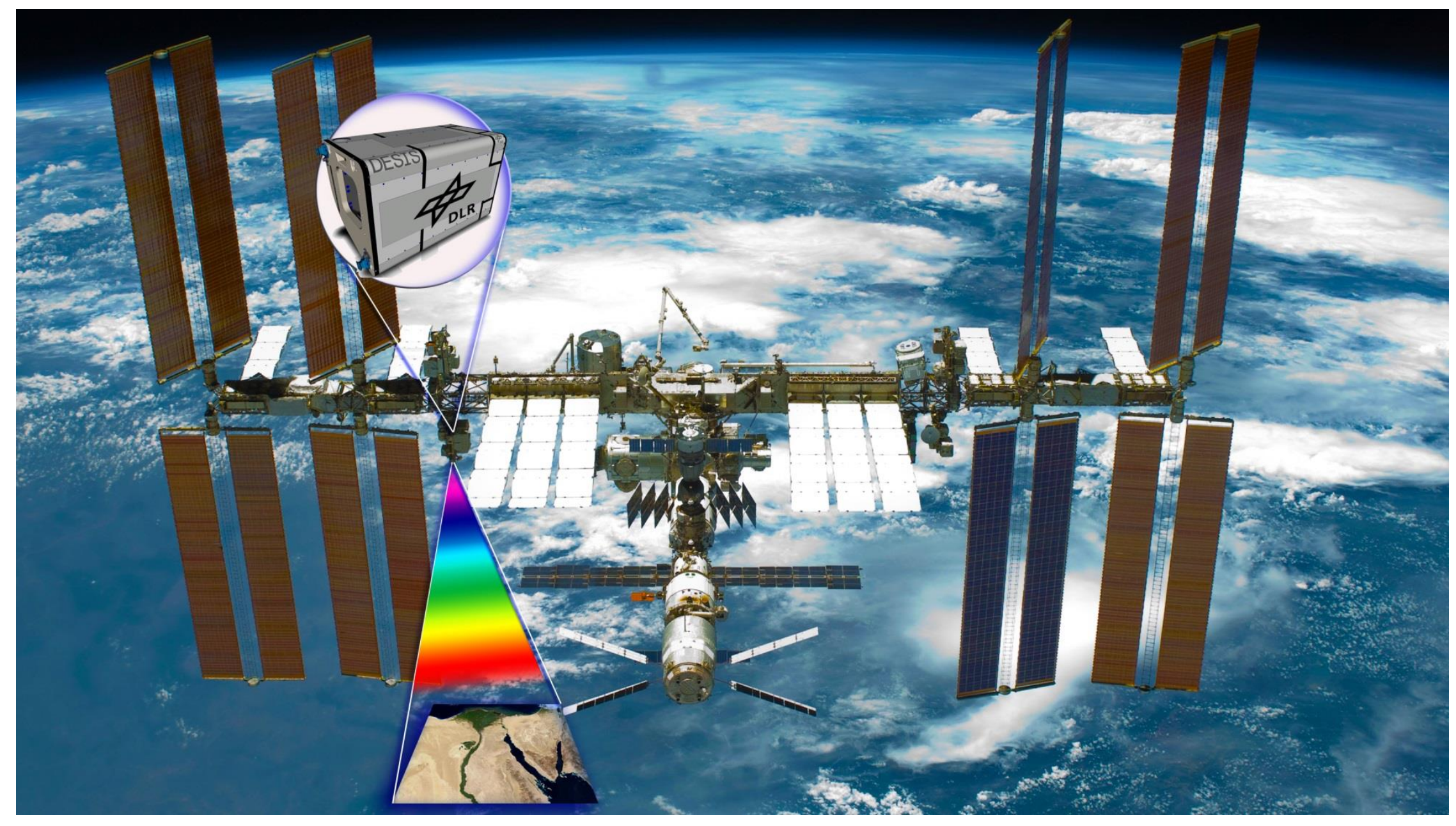




\section{DESIS: DLR Earth Sensing Imaging Spectrometer}

- MUSES: Multiple User System for Earth Sensing

- Commercial imaging platform in cooperation with Teledyne Brown Engineering

- International Space Station (ISS)

$\Rightarrow$ height appr. $400 \mathrm{~km}$

- Push-Broom Scanner: 30 km swath width

- Resolution:

$30 \mathrm{~m}$

- Spectral channels:

235

- Spectral range:

$400 \mathrm{~nm} \ldots 1000 \mathrm{~nm}$

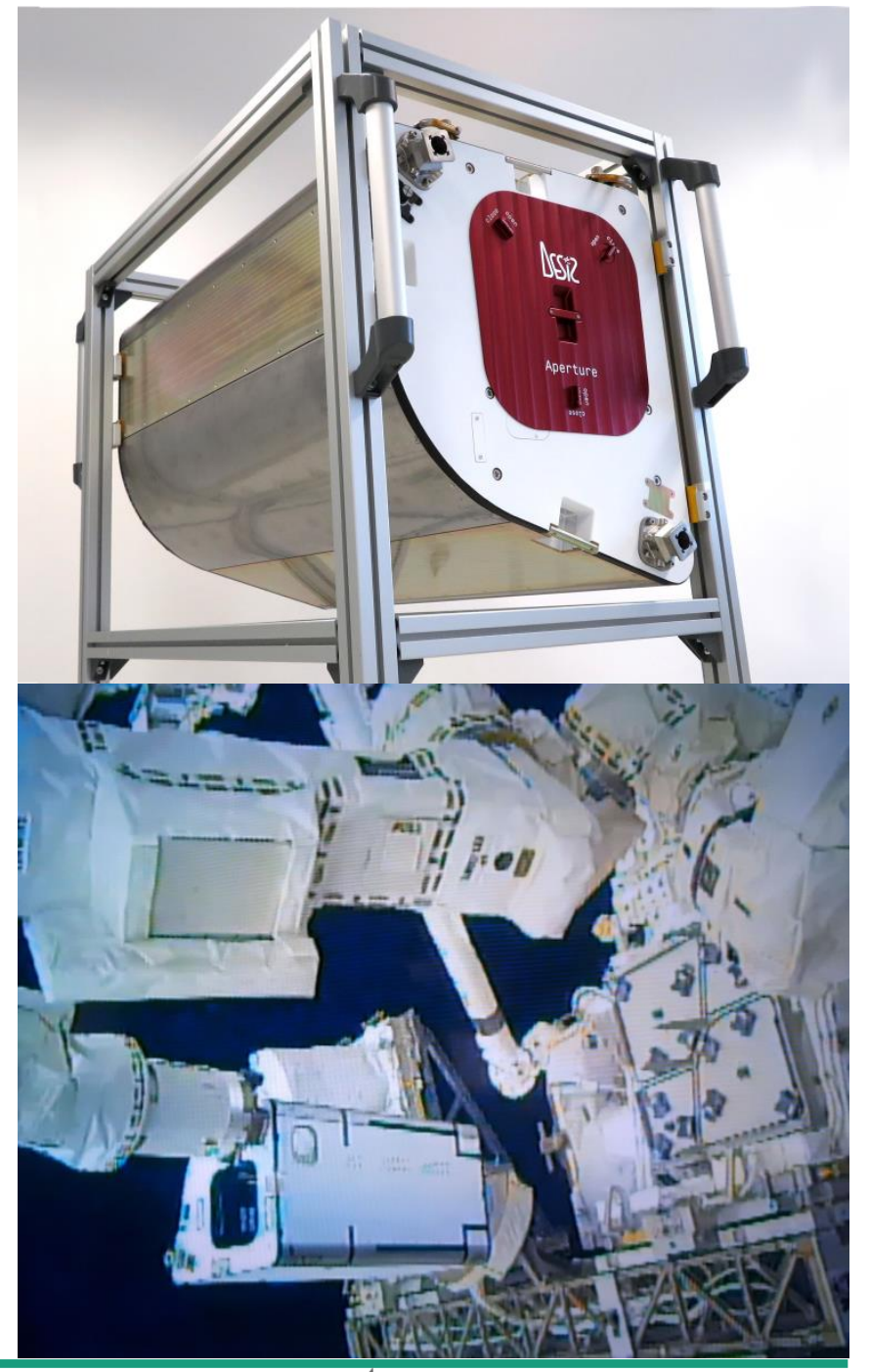




\section{Optical and mechanical concept}

- Three-Mirror-Anastigmat (TMA)

- Field: $\quad \pm 2.2^{\circ}$ across track

- 3 aspheres on common axis

- Focal length: $320 \mathrm{~mm}$

- Aperture: F/2.8

- Slit

- Offner-Type grating spectrometer

- Free-form surface on large mirror $\Rightarrow$ reduce wavelength dependent astigmatism

- Small spherical mirror with binary grating grating

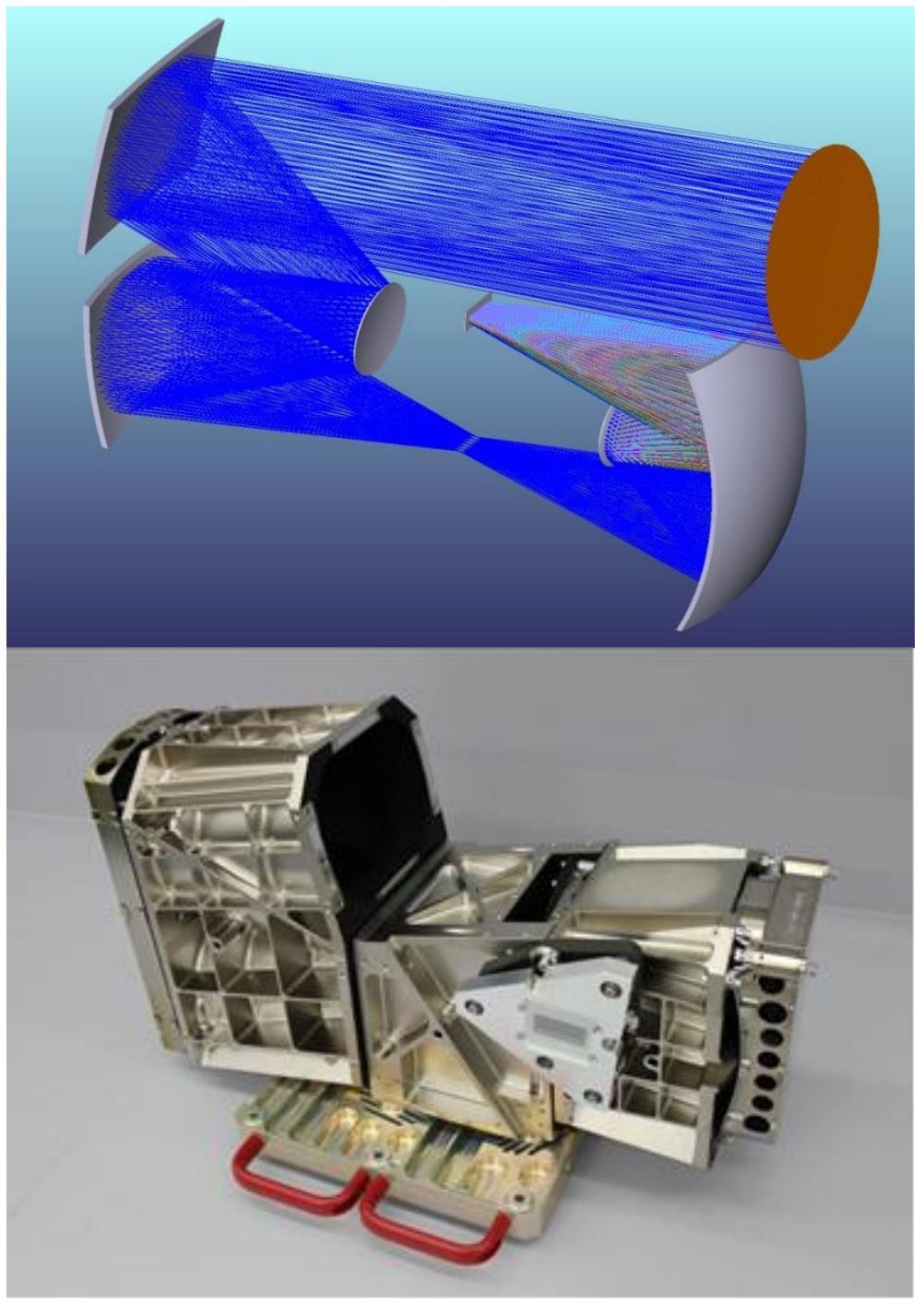




\section{Outline}

- Introduction

- Components and pre-integration

- Instrument integration and results

- Summary 


\section{Integration concept: Prealignment}

- Mounting of sub-assemblies (mirrors, slit)

- Reference pins and bars on housing used for reproducible alignment of the optical elements (using height gauges)

- References on housing made by SPD fly-cutting

$\Rightarrow$ match reference features on the mirrors

$\Rightarrow$ reference for interferometer

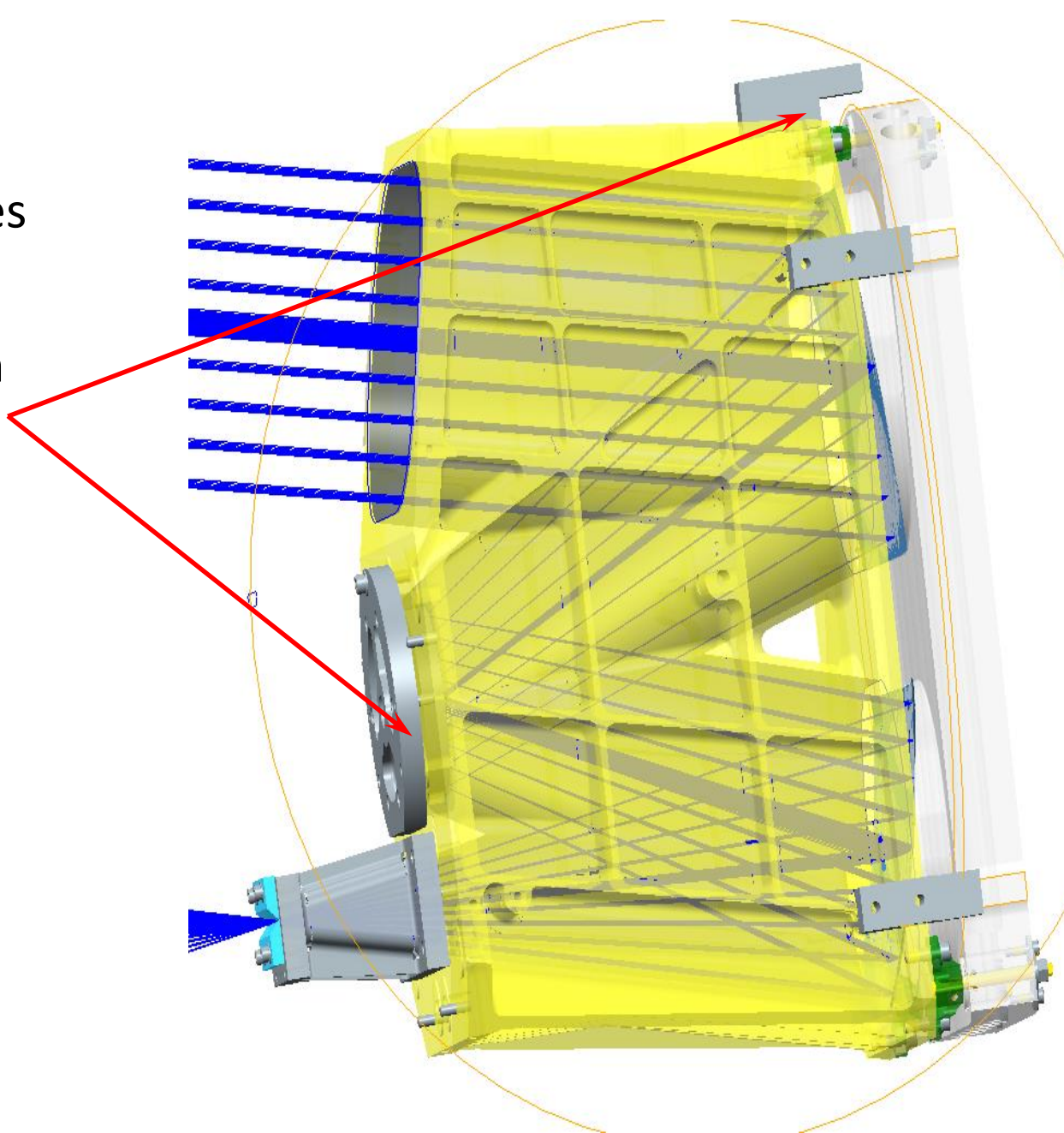




\section{Process chain for the manufacturing of metal mirrors}

\section{for NIR \& IR applications vs. VIS \& UV applications}

\section{Surface requirements IR}

- Roughness: < $20 \mathrm{~nm}$ rms

- Figure: $\quad<2 \mu \mathrm{m}$ p.-v.

Surface requirements VIS

- Roughness: < 2 nm rms

- no periodical structures

- Figure: <200nm p.-v.

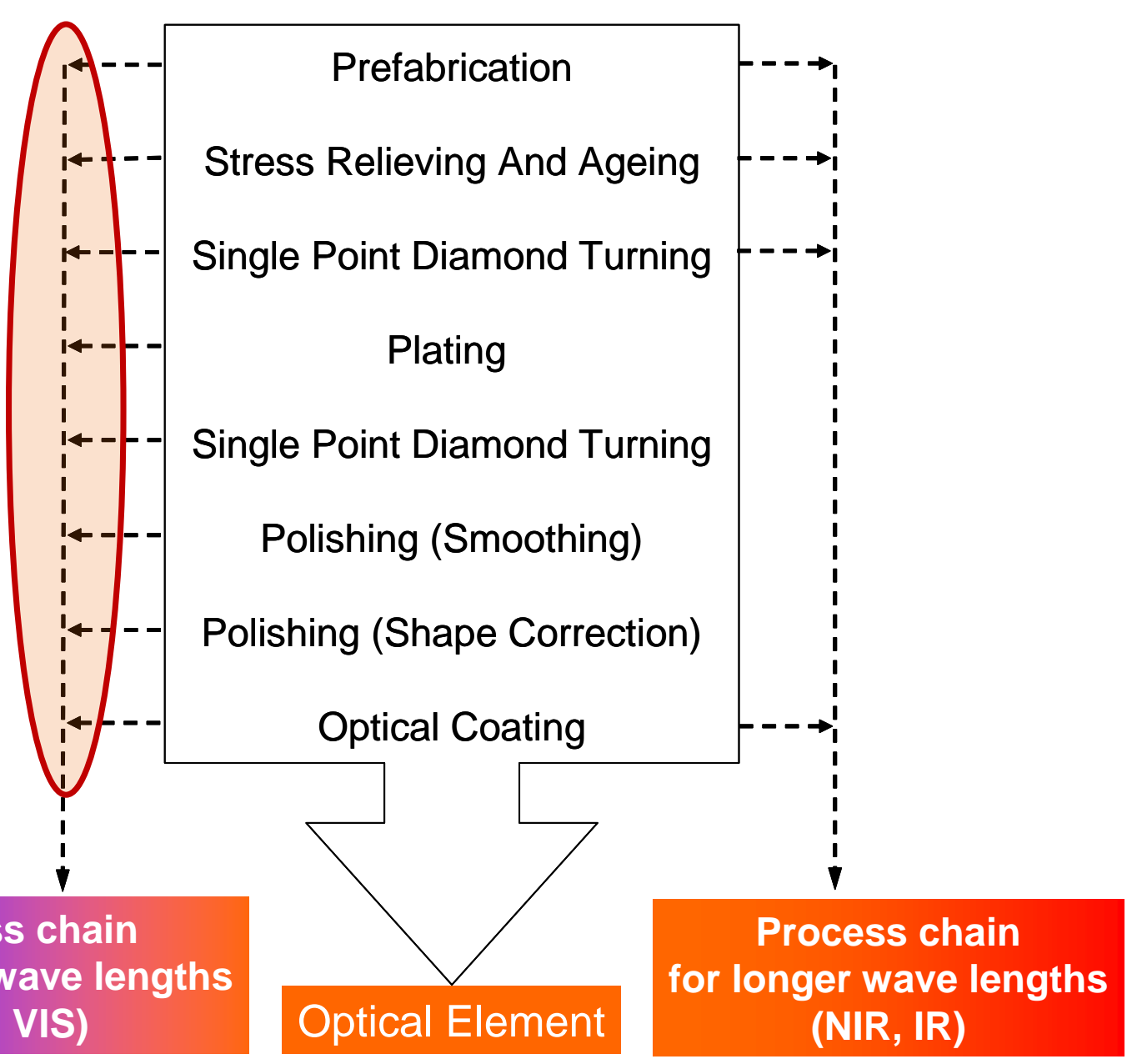

Fraunhofer
Optical Element
Process chain

for shorter wave lengths

(UV, VIS) 


\section{Mirror design M1/M3 subassembly}

- M1 and M3 on a common substrate

$\Rightarrow$ tight tolerances for mirror pos.

$\Rightarrow$ freeform manufacturing

- Manufacturing of mounting and reference structures in the same machining run

$\Rightarrow$ common coordinate system

$\Rightarrow$ simplified testing interface to $\mathrm{CGH}$

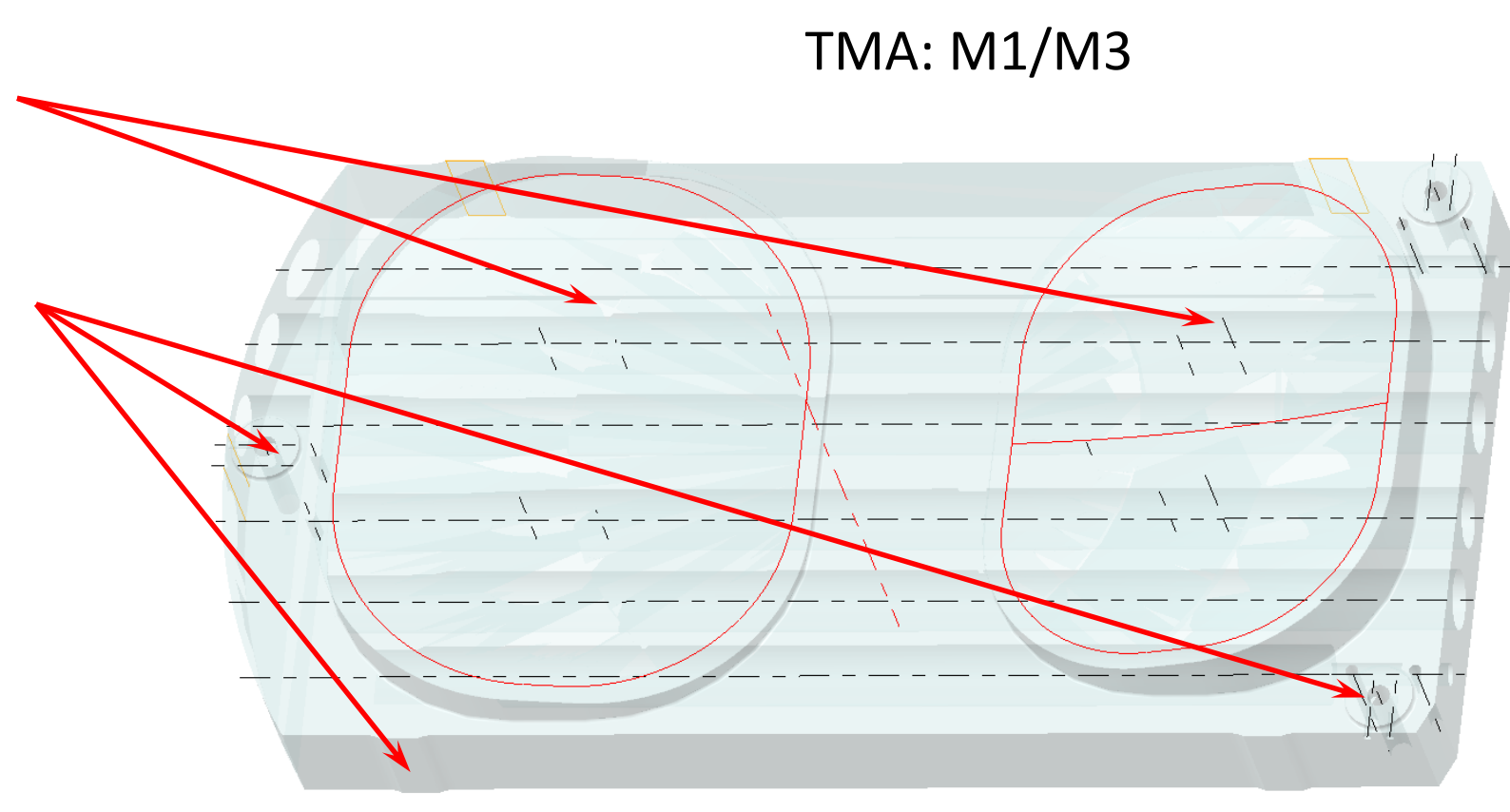

More details: M. Beier, Session 4b 


\section{Results for the TMA mirrors}

- Interferometric test of surface quality after coating

\begin{tabular}{|l|c|l|l|}
\hline Mirror & p.-v. $[\mathrm{nm}]$ & r.m.S. [nm] & Power [nm] \\
\hline M1 & 291 & 42 & -76 \\
\hline M2 & 37 & 5 & 315 \\
\hline M3 & 110 & 16 & 154 \\
\hline
\end{tabular}




\section{Grating}

- Metal-based technology similar to the processing of the mirrors:

AlSi40+NiP

- Grating formed by single point diamond fly-cutting: period: $13.4 \mu \mathrm{m}$ grove depth:

- Binary grating with a duty cycle of 1:1 (suppression of $2^{\text {nd }}$ order) $1^{\text {st }}$ order efficiency: $(32.5 \pm 2.5) \%$ at $633 \mathrm{~nm}$

- Remaining form error: 36 nm r.m.s.
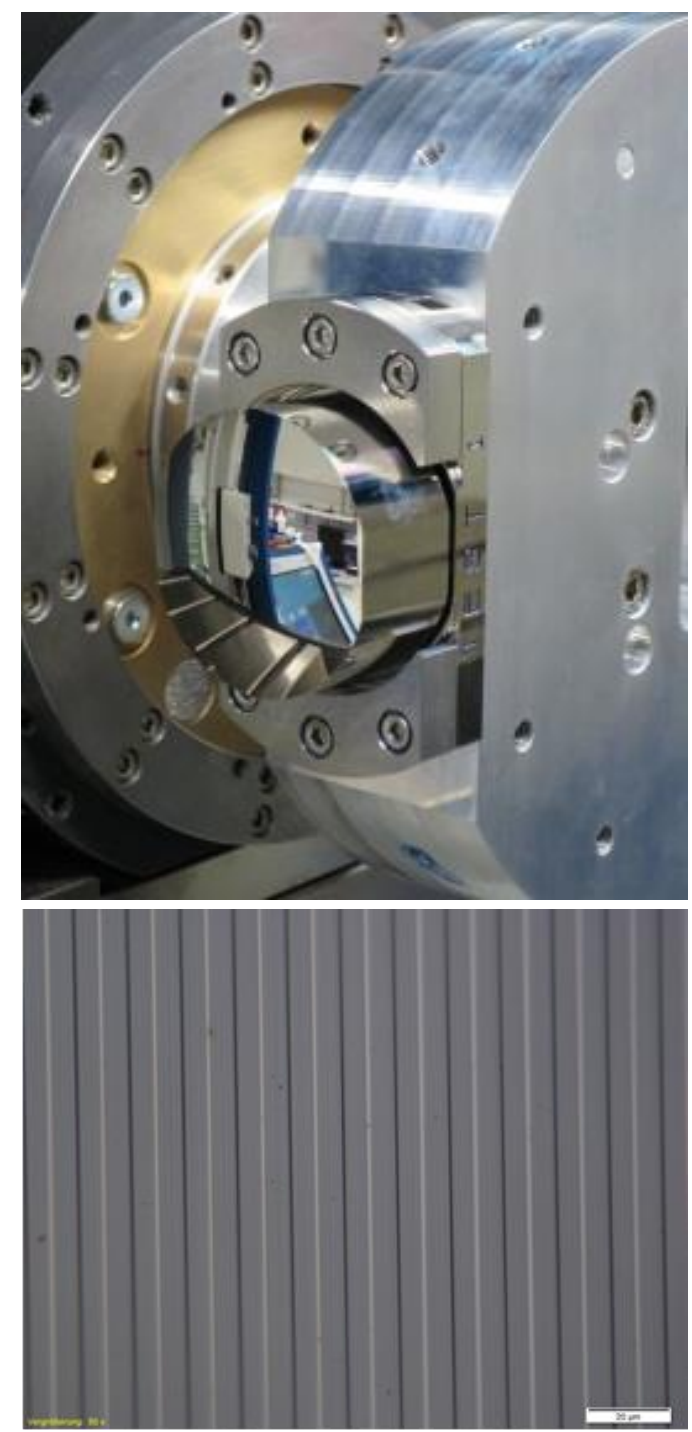


\section{Slit}

- Metal-based technology similar to the processing of the mirrors: AlSi40+NiP

- Blades formed by single point diamond fly-cutting including reference surfaces for mounting and metrology Blade straightness: $\quad 1 \mu \mathrm{m}$

- Manual adjustment using height gauges and camera control: Slit width:

$23.9 \mu \mathrm{m}$

Width variation:

$0.5 \mu \mathrm{m}$

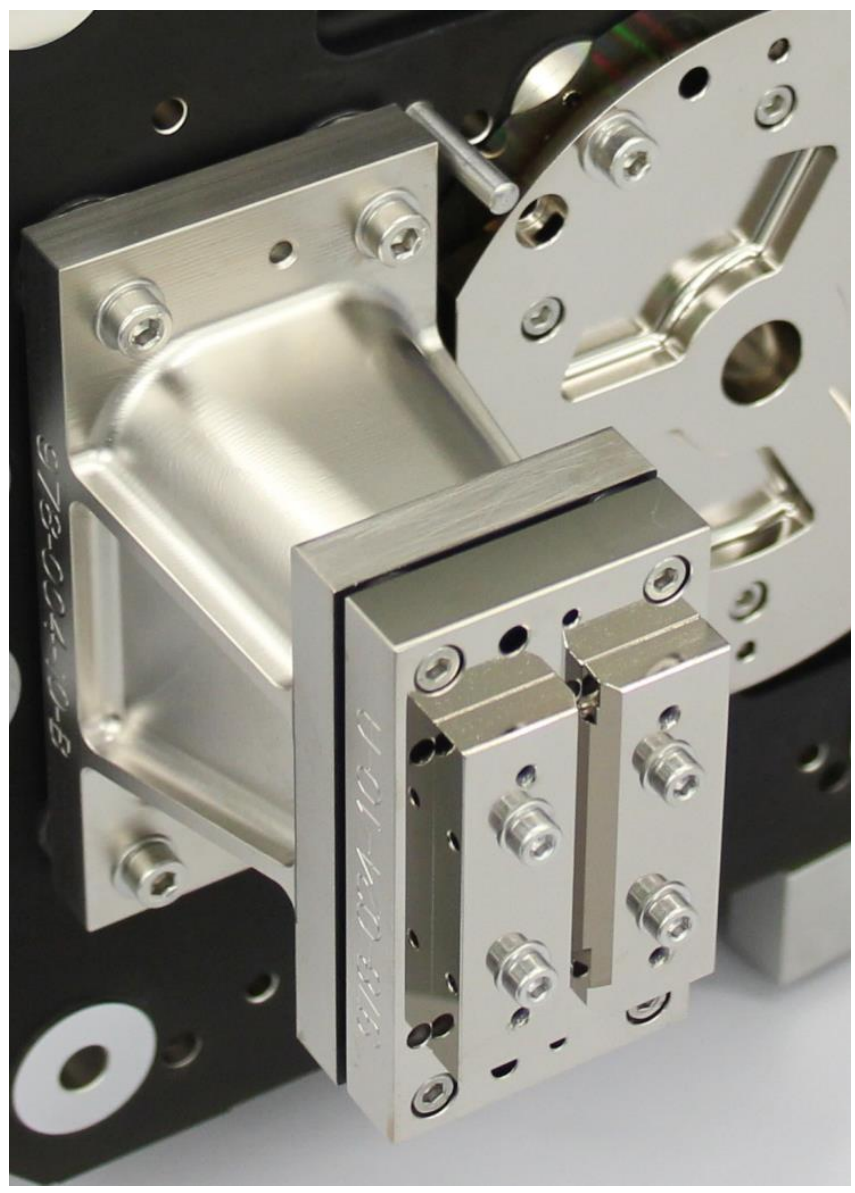




\section{Outline}

- Introduction

- Components and pre-integration

- Instrument integration and results

- Summary 


\section{TMA final alignment}

- On hexapod in front of interferometer

- Double-pass: plane input, reference sphere in image pos.

- Interferometric image imediately after prealignment $\Rightarrow$ Fast alignment

- 35 nm r.m.s.in central field $<80 \mathrm{~nm}$ at edges of the slit
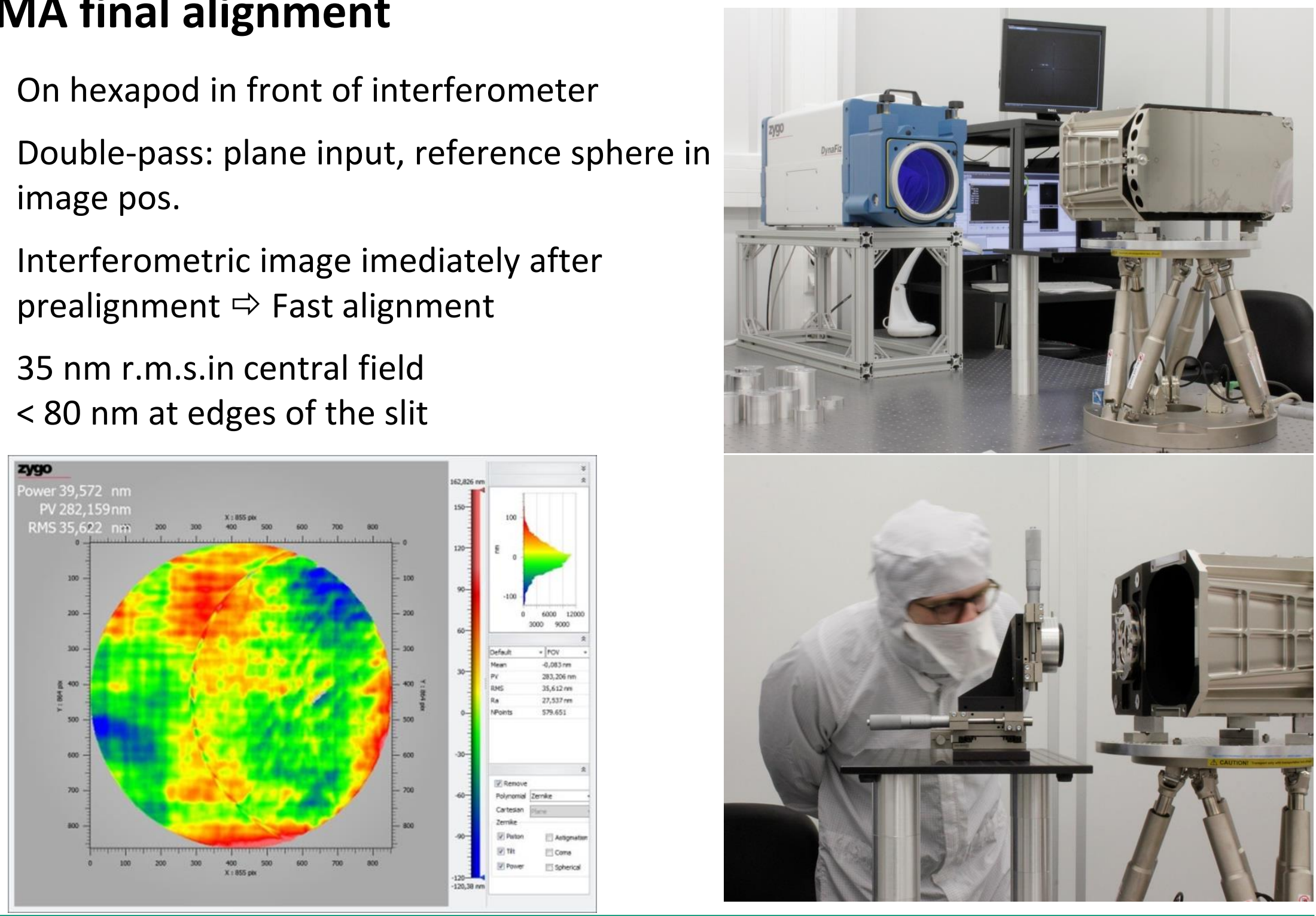


\section{Spectrometer integration}

- Similar integration+alignment strategy

- Double-pass: spherical input, reference sphere in image pos.

- 80 nm r.m.s.in central field $<115 \mathrm{~nm}$ at edges of the slit

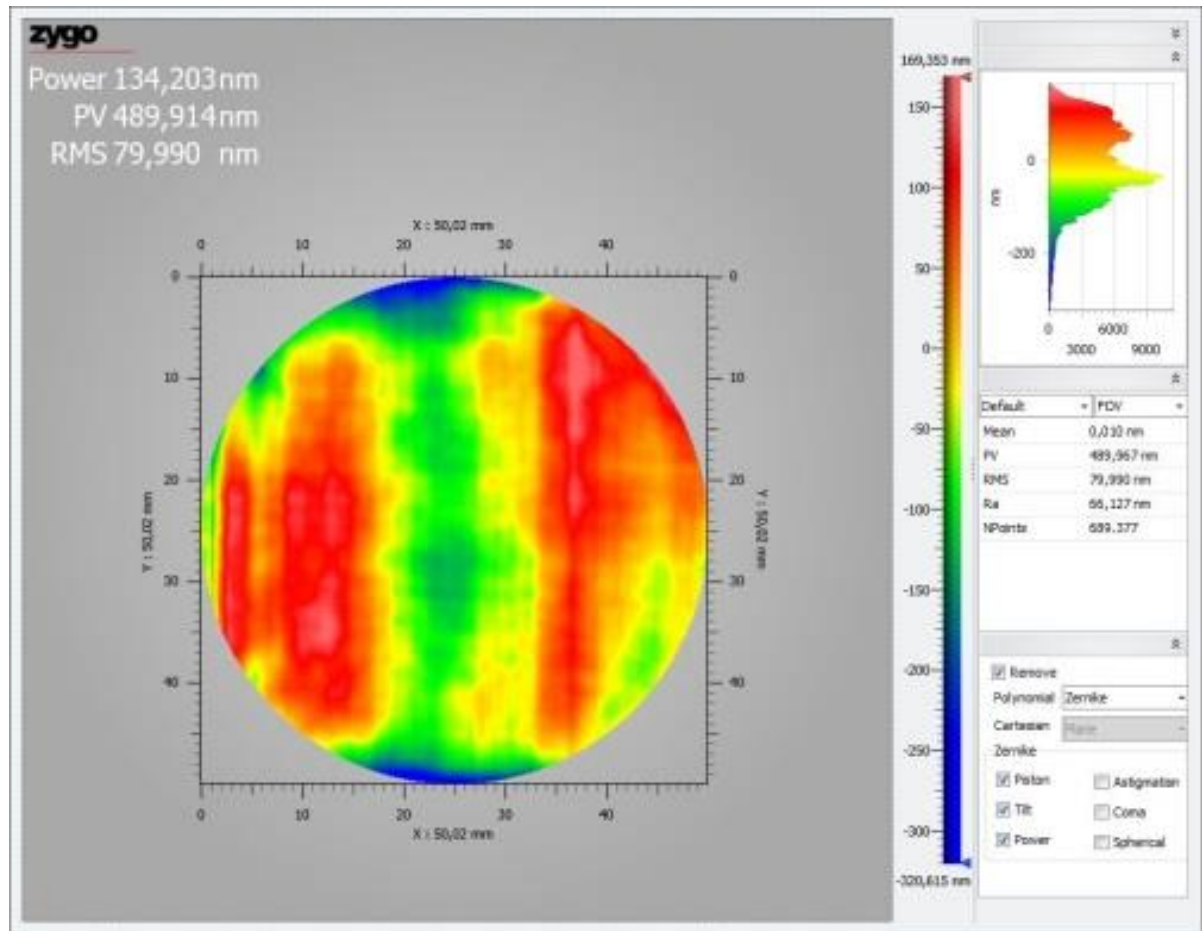

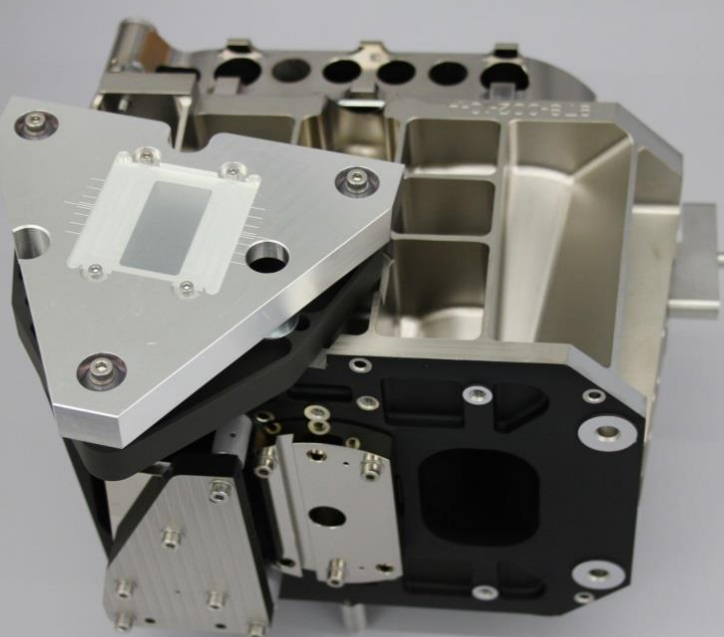

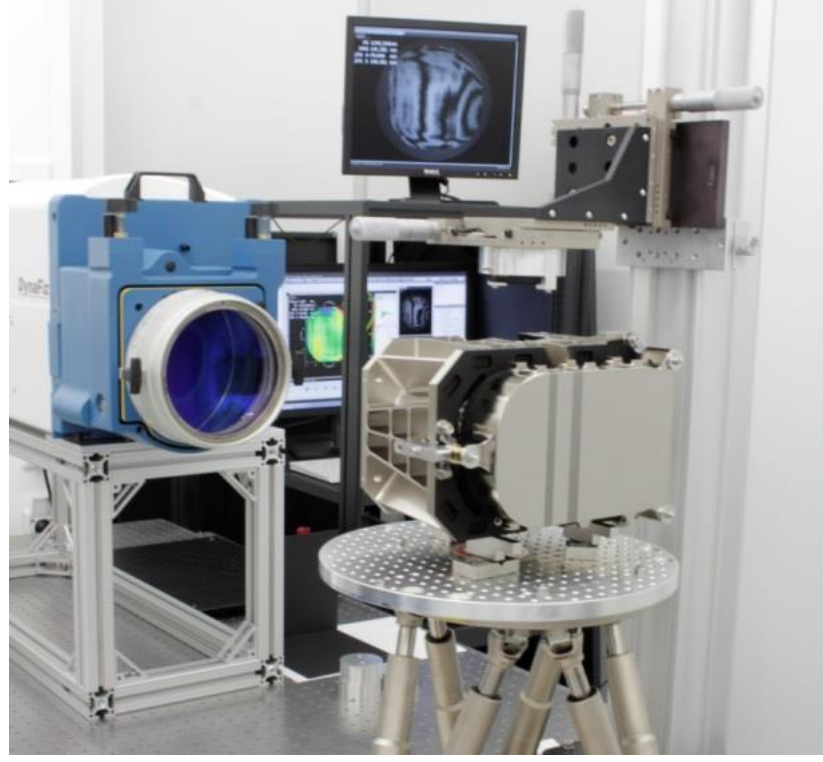

44 Fraunhofer 


\section{Integration of the optical system}

- Assemble subsystems using references and height gauges

- Double-pass interferometric measurement: plane input, reference sphere in image pos.

- Interferometric test at 9 field points:

$0^{\circ}, \pm 0.75^{\circ}, \pm 1.5^{\circ}, \pm 2.0^{\circ}, \pm 2.2^{\circ}$

- Remaining wave front error: 63 ... $120 \mathrm{~nm}$ r.m.s. at $633 \mathrm{~nm}$

$\Rightarrow$ withint expectations from optical tolerancing

- $\Rightarrow$ Fulfills system requirements
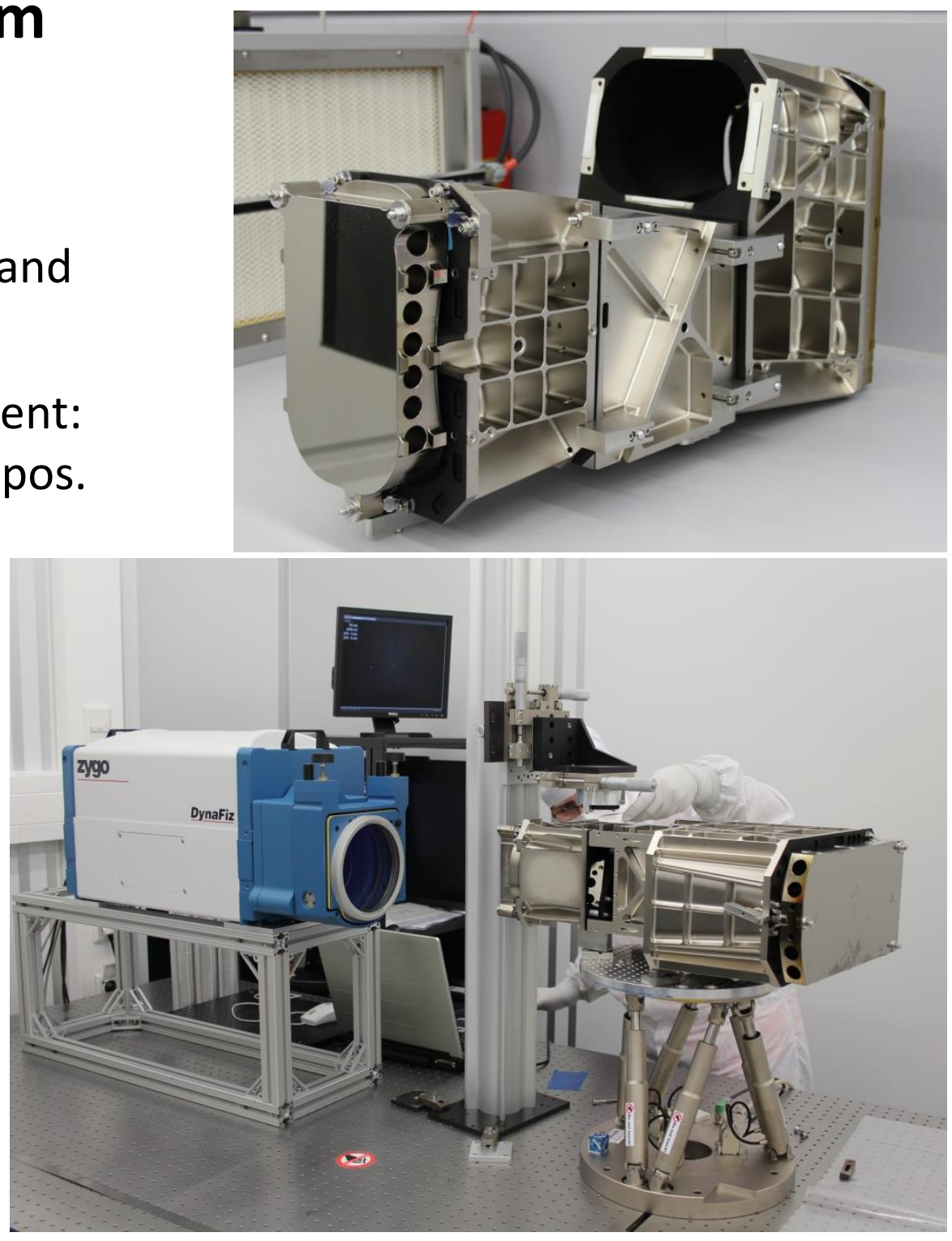


\section{Results}

\begin{tabular}{|c|c|c|}
\hline Entrance angle $\left[^{\circ}\right]$ & $\begin{array}{l}\text { Wavefront } \\
\text { error } \\
\text { rms [nm] }\end{array}$ & $\begin{array}{l}\text { Wavefront } \\
\text { error } \\
\mathrm{PV}[\mathrm{nm}]\end{array}$ \\
\hline-2.2 & 116 & 771 \\
\hline-2.0 & 119 & 755 \\
\hline-1.5 & 116 & 734 \\
\hline-0.75 & 98 & 570 \\
\hline 0 & 83 & 532 \\
\hline+0.75 & 68 & 541 \\
\hline+1.5 & 63 & 400 \\
\hline+2.0 & 78 & 579 \\
\hline+2.2 & 96 & 712 \\
\hline
\end{tabular}




\section{Summary}

- Optical system for an imaging spectrometer has been realized and fulfills the performance requirements

- All-metal design:

- Mirror manufacturing: AlSi42+NiP (CTE matched)

- Free-form manufacturing

- AlSi42 also used for instrument housing

- Grating on curved substrate by single-point diamond fly-cutting

- Slit

- „Snap-in “ integration:

- Integrated alignment features (by SPDT)

- Initial alignment via mechanical stops

- Final alignment and testing with interferometer mechanical stops used for reproducible motion 
- Optical system has been transferred to DLR in July 2017 for integration

- DESIS has been launched successfully on June 29, 2018

- Mounted onto MUSES

- First images on Aug. 31, 2018
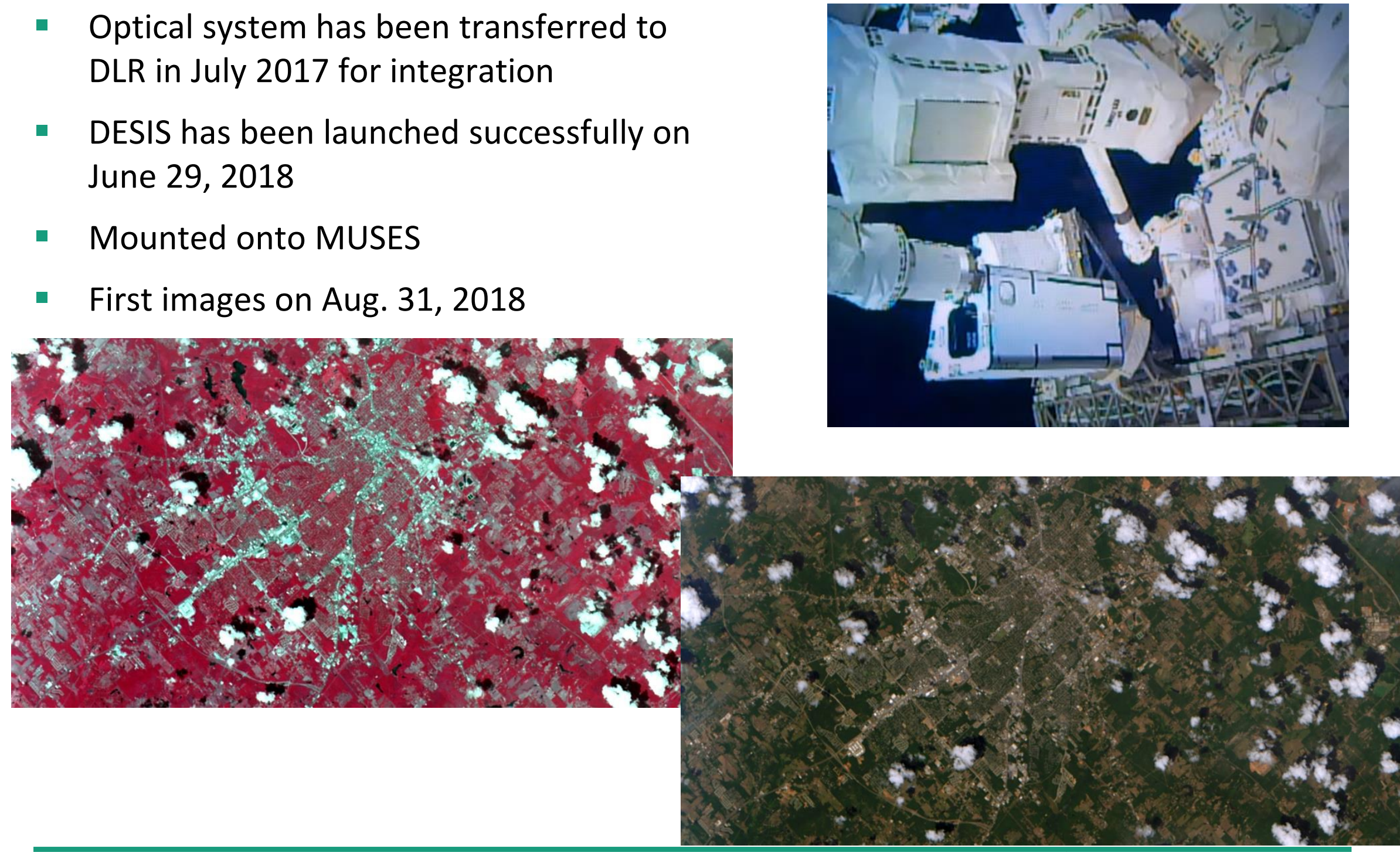


\section{Thank you for your attention!}

We acknowledge funding of basic research used for the presented application by German Aerospace Center (DLR) within the projects IRS-ServoTech and VISTEL under grant numbers 50EE1006 and 50EE1224, respectively.

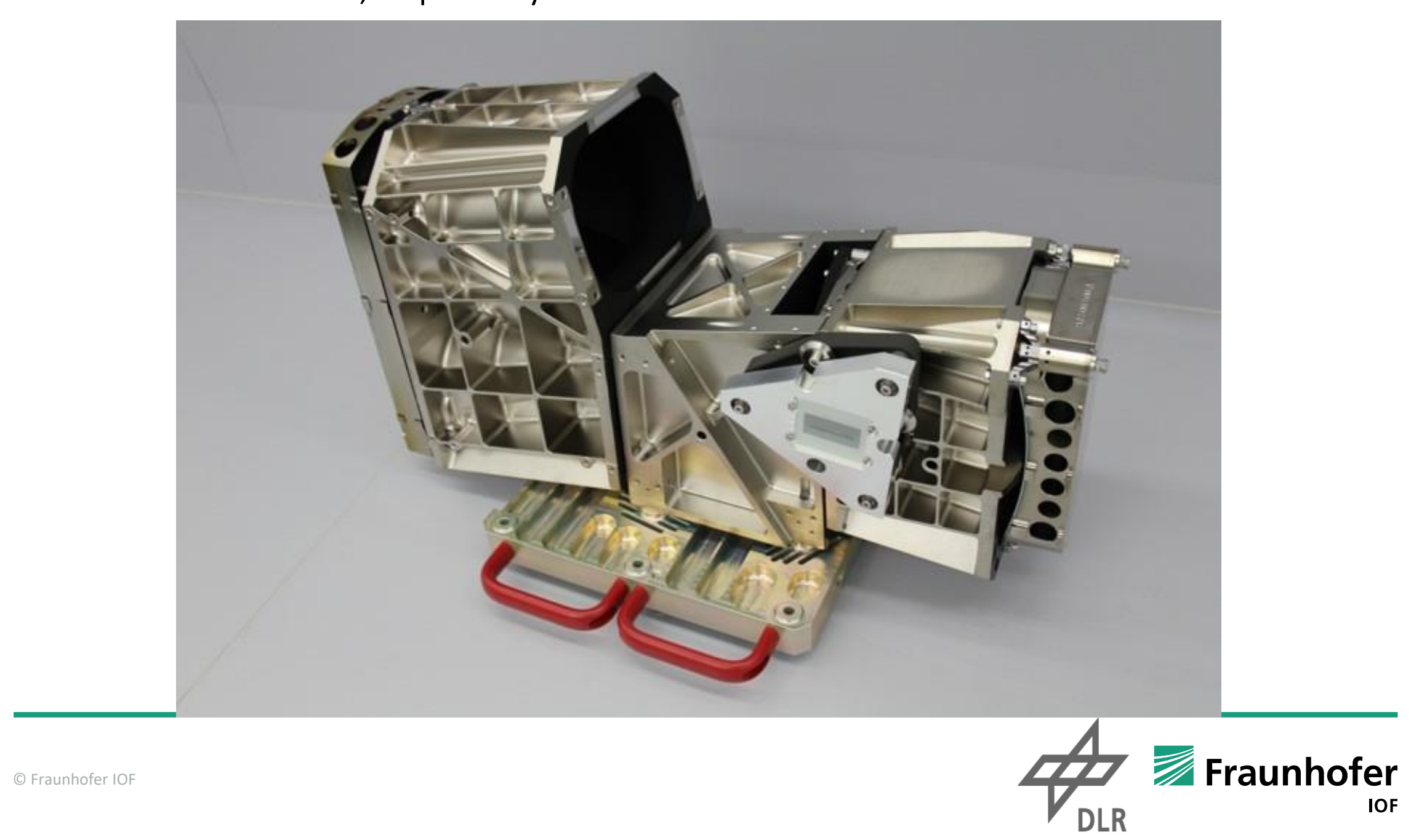

